\title{
Regioselective enzymatic synthesis of estradiol 17-fatty acid esters
}

\author{
Eduardo M. Rustoy, Ignacio E. Ruiz Arias, and Alicia Baldessari* \\ Departamento de Química Organica y UMYMFOR, \\ Facultad de Ciencias Exactas y Naturales, \\ Universidad de Buenos Aires, Pabellón 2, Piso 3, Cuidad Universitaria, 1428, Buenos Aires, \\ Argentina, \\ E-mail: alib@qo.fcen.uba.ar
}

\section{Dedicated to Professor Rosa M. de Lederkremer on her $70^{\text {th }}$ aniversary}

\begin{abstract}
A series of acyl esters of 3,17- $\beta$-estradiol has been prepared by an enzymatic methodology. Eleven 17-monoacyl products (five novel compounds) were obtained in a highly regioselective way by acylation of 3,17- $\beta$-estradiol or by alcoholysis of the corresponding diacyl derivatives. The influence of various reaction parameters such as molar ratio, enzyme:substrate ratio and temperature was evaluated. Among the tested lipases, Candida rugosa lipase appeared to be the most appropriate in monoacylation and lipase from Candida antarctica in alcoholysis. The advantages presented by this methodology such as mild reaction conditions, economy and low environmental impact, make the biocatalysis a convenient way to prepare monoacyl derivatives of 3,17- $\beta$-estradiol containing the aromatic 3-hydroxyl group free. Some of these compounds are recongnized as useful products in the pharmaceutical industry.
\end{abstract}

Keywords: Lipases. enzymatic acylation and alcoholysis. 3,17- $\beta$-estradiol esters

\section{Introduction}

Human estrogens, such as 3,17- $\beta$-estradiol 1, are known to exert powerful antioxidant effects in lipid-aqueous systems in vitro. ${ }^{1-3}$ The antioxidant efficacy appears to depend on unsubstituted hydroxyl groups on the aromatic ring A of estrogen molecules, ${ }^{4-7}$ a structure similar to that seen in other natural antioxidants such as $\alpha$-tocopherol $\mathbf{2}$ and several isoflavone phytoestrogens as well as flavones (Figure 1). ${ }^{8}$ 


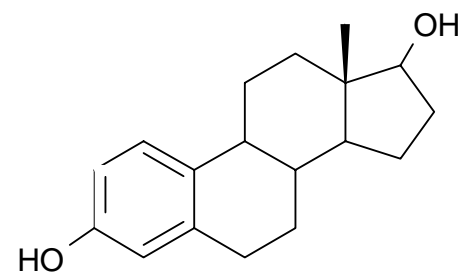

1

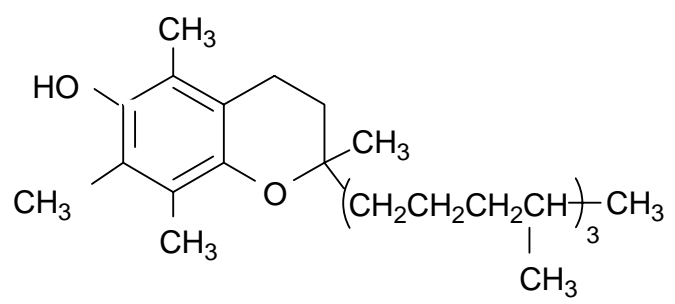

2

\section{Figure 1}

The identification of estradiol esters formed by lecithin:cholesterol acyltransferase ${ }^{9}$ and the esterification site of estradiol at C-17 in the D ring, ${ }^{10}$ suggested that these derivatives might become structural components in lipoproteins. These lipoprotein-associated molecules would be active possibly interfering with the atherosclerotic process.

Fatty acid esterification of steroid hormones is a metabolic transformation which prolongs and potentiates hormonal activity, although the esters are prohormones which require hydrolytic cleavage for their activation. ${ }^{11}$ In theory, estrogen fatty acid esters could constitute a hormonal reservoir mainly residing in fat tissue. ${ }^{12}$ One may speculate that specific esterases might become activated and liberate the estrogens at times when steroidogenic organs become quiescent or lose their function. ${ }^{11}$

Although there is mounting evidence supporting important functions for steroid esters, all the functions remained speculative and further studies are of particular interest. Then, fatty acid monesters of 3,17- $\beta$-estradiol in the position 17 of ring $\mathrm{D}$ could be useful to ascertain the possible utility of estrogens as protective against atherosclerosis and to provide standards for metabolic studies.

The regioselective tranformation of polyfunctional compounds such as steroids, is a challenging problem in organic synthesis, especially in case of a structure sensitive to acid, base, oxidation, or reduction, which limits the choice of reagents needed to accomplish a particular transformation. In recent years, lipases have become attractive as biocatalysts for chemo-, regioand stereoselective reactions under mild conditions. ${ }^{13,14}$ They can be used in a wide variety of organic solvents and do not require a coenzyme for activity. ${ }^{15,16}$

Specifically in the steroids field, enzyme catalysis can play an important role for the mild and selective interconversion of functional groups via regioselective transformations. ${ }^{17-21}$ Studies carried out in our laboratory on the esterification and transesterification of polyfunctionalyzed steroids, have shown that lipases can act on substituents either on A-ring or on the D-ring. ${ }^{22,23}$ Thus, in previous papers we observed that, in androstanes and pregnanes, Candida rugosa lipase showed a preference for C-3 hydroxyl or acyloxy groups, whereas Candida antarctica catalyzed the reactions in D-ring. ${ }^{24,25}$

Since 17-monoacyl esters of 3,17- $\beta$-estradiol are biologically active compounds used as "long acting" estrogens, a simple and selective procedure to obtain them is required. In 
continuation of our work on enzymatic transformation of steroids, we report in the present paper the results obtained in lipase-catalyzed acylation of 3,17- $\beta$-estradiol $\mathbf{1}$ and alcoholysis of its dioleoyl derivative.

\section{Results and Discussion}

We have prepared, under mild reaction conditions monoacylated derivatives of 3,17- $\beta$-estradiol in a regioselective way and in high yields. The different compounds have been obtained through acylation of the substrate or alcoholysis of the oleyl derivative using lipases from several sources as catalysts.

\section{Enzymatic acylation}

The enzyme-catalyzed acylation let to obtain, in a regioselective way, monoacylated derivatives with the acyl group exclusively suited in the 17 position of ring D in the steroidal skeleton (Scheme 1).

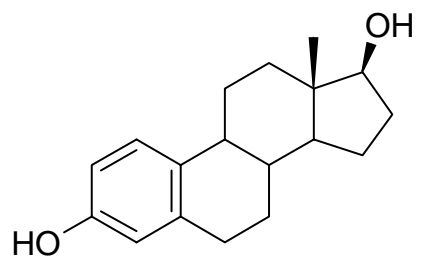

1

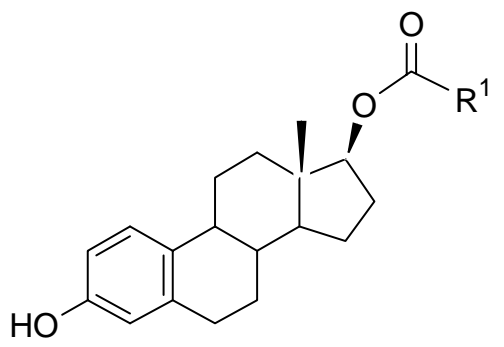

3

\begin{tabular}{|c|c|c|}
\hline 3a & $\mathrm{R}^{1}=\mathrm{CH}_{3}\left(\mathrm{CH}_{2}\right)_{3} \mathrm{CO}-$ & $\mathrm{R}^{2}=\mathrm{CH}_{3} \mathrm{CH}_{2}$ \\
\hline 3a & $\mathrm{R}^{1}=\mathrm{CH}_{3}\left(\mathrm{CH}_{2}\right)_{3} \mathrm{CO}-$ & $\mathrm{R}^{2}=\mathrm{H}$ \\
\hline $3 \mathbf{b}$ & $\mathrm{R}^{1}=\mathrm{CH}_{3}\left(\mathrm{CH}_{2}\right)_{4} \mathrm{CO}-$ & $\mathrm{R}^{2}=\mathrm{H}$ \\
\hline 3d & $\mathrm{R}^{1}=\mathrm{CH}_{3}\left(\mathrm{CH}_{2}\right)_{8} \mathrm{CO}-$ & $\mathrm{R}^{2}=\mathrm{H}$ \\
\hline 3d & $\mathrm{R}^{1}=\mathrm{CH}_{3}\left(\mathrm{CH}_{2}\right)_{10} \mathrm{CO}-$ & $\mathrm{R}^{2}=\mathrm{H}$ \\
\hline $3 \mathbf{e}$ & $\mathrm{R}^{1}=\mathrm{CH}_{3}\left(\mathrm{CH}_{2}\right)_{12} \mathrm{CO}-$ & $\mathrm{R}^{2}=\mathrm{H}$ \\
\hline $3 f$ & $\mathrm{R}^{1}=\mathrm{CH}_{3}\left(\mathrm{CH}_{2}\right)_{14} \mathrm{CO}-$ & $\mathrm{R}^{2}=\mathrm{H}$ \\
\hline $3 g$ & $\mathrm{R}^{1}=\mathrm{CH}_{3}\left(\mathrm{CH}_{2}\right)_{16} \mathrm{CO}-$ & $\mathrm{R}^{2}=\mathrm{H}$ \\
\hline $3 \mathbf{h}$ & $\mathrm{R}^{1}=\mathrm{CH}_{3}\left(\mathrm{CH}_{2}\right)_{7} \mathrm{CH}=\mathrm{CH}\left(\mathrm{CH}_{2}\right)_{7} \mathrm{CO}-$ cis & $\mathrm{R}^{2}=\mathrm{H}$ \\
\hline $3 \mathbf{i}$ & $\mathrm{R}^{1}=\mathrm{CH}_{3}\left(\mathrm{CH}_{2}\right)_{7} \mathrm{CH}=\mathrm{CH}\left(\mathrm{CH}_{2}\right)_{7} \mathrm{CO}$ - trans & $\mathrm{R}^{2}=\mathrm{H}$ \\
\hline $3 \mathbf{j}$ & $\mathrm{R}^{1}=\mathrm{CH}_{3}\left(\mathrm{CH}_{2}\right)_{3}\left(\mathrm{CH}_{2} \mathrm{CH}=\mathrm{CH}\right)_{2}\left(\mathrm{CH}_{2}\right)_{7} \mathrm{CO}-$ cis, cis & $\mathrm{R}^{2}=\mathrm{H}$ \\
\hline $3 \mathbf{k}$ & $\mathrm{R}^{1}=\mathrm{CH}_{3}\left(\mathrm{CH}_{2} \mathrm{CH}=\mathrm{CH}\right)_{3}\left(\mathrm{CH}_{2}\right)_{7} \mathrm{CO}-$ cis, cis, cis & $\mathrm{R}^{2}=\mathrm{H}$ \\
\hline
\end{tabular}

\section{Scheme 1}




\section{1.a. Enzyme screening and solvent effect}

Several commercial lipases in different solvents were evaluated in the acylation reaction of $\mathbf{1}$ with oleic acid: Candida rugosa lipase (CRL), Candida antarctica lipase B (CAL B); Lipozyme from the fungus Rizomucor miehei (LIP), pancreatin, porcine pancreatic lipase (PPL) and lipase from Pseudomonas sp. (PS-C). The solvents selected were acetone, $t$-butanol, $t$-amyl alcohol, diisopropyl ether and toluene. Reactions were carried out at $33^{\circ} \mathrm{C}$ using an enzyme: substrate ratio of 5 and acylating agent: estradiol ratio of 2.5. TLC monitoring allowed the identification of the lipase able to promote the monoacylation of $\mathbf{1}$.

In the absence of biocatalyst no product was obtained and, among the tested lipases, only CRL gave satisfactory results. GC/MS analysis of the CRL-catalyzed esterification in toluene showed that this lipase was completely regioselective obtaining exclusively the oleyl derivative in 17 position $\mathbf{3 h}$ in $62 \%$ yield, whereas in diisopropylether a mixture of $\mathbf{3 h}$ and isopropyl oleate was obtained.

Working in the above mentioned reaction conditions, it is known that microbial lipases accept equatorial $3 \beta-\mathrm{OH}$ (PSL, CRL) or axial $3 \alpha-\mathrm{OH}$ (CAL B) of ring $\mathrm{A}$ in the steroidal skeleton, but none of the lipases tested in this case was active in acylation of the aromatic 3hydroxyl group in estradiol in the presence of solvents.

The application of lipases let to obtain 17-monooleyl $\mathbf{3 h}$ derivative with a high degree of selectivity, achieving a remarkable improve to the chemical approach. According to literature it is not possible to obtain exclusively the product $\mathbf{3 h}$. The chemical acylation of $\mathbf{1}$ with oleyl chloride in pyridine at $0^{\circ} \mathrm{C}$ affords a mixture of products: a major product which is the 3,17 diester and two minor products corresponding to the C-3 monoester and C-17 monoester, as well as free estradiol. ${ }^{26,27}$

Considering the results of enzymatic screening, we observed that CRL was the enzyme of choice for the synthesis of 3,17- $\beta$-estradiol-17-oleate $\mathbf{3 h}$ using toluene as solvent and was then selected for any further experiments.

\section{1.b. Effect of nature of the acyl donor}

Using CRL in toluene we investigated the influence of the acyl donor on the reaction yield. Estradiol esterification with oleic acid was compared to transesterification using ethyl oleate as acyl donor. We observed that esterification with oleic acid was much better with more than $75 \%$ formation of $\mathbf{3 h}$ while the use of ethyl ester gave very poor yield not exceeding 39\% after $96 \mathrm{~h}$. Interestingly, the same results were also reported by us in enzymatic acylation of pyridoxine ${ }^{28}$ and dehydroepiandrosterone ${ }^{23}$ and other authors in the preparation of cholesterol- ${ }^{29}$ and phytosterols oleate. ${ }^{30}$ Therefore, in all further experiments with CRL, esterification processes were chosen using free fatty acids as acyl donors.

\section{1.c. Effect of acylating agent : substrate ratio}

The effect of the influence of acylating agent: substrate ratio on reaction yield was evaluated in the esterification of estradiol 1 with oleic acid in toluene using CRL. Reaction courses were 
followed by GC at 24 and $72 \mathrm{~h}$ of reaction. As expected, it was observed that a molar excess of fatty acid was advantageous for the reaction (Table 1) with 2.5:1 oleic acid/ estradiol ratio giving the best results ( $78 \%$ esterification degree after $72 \mathrm{~h}$, entry 2$)$. A higher excess of fatty acid did not improve yields in comparison with 2.5 ratio.

Table 1. Acylating agent/substrate ratio on the CRL-catalyzed synthesis of 3,17- $\beta$-estradiol-17oleate $\mathbf{3 h}$

\begin{tabular}{cccc}
\hline \multirow{2}{*}{ Entry } & $\mathrm{A} / \mathrm{S}$ & \multicolumn{2}{c}{${\text { Yield }(\%)^{\mathrm{a}}}^{\mathrm{a}}$} \\
\cline { 3 - 4 } & $1: 1$ & 34.6 & $72 \mathrm{~h}$ \\
\hline 1 & $2.5: 1$ & 65.7 & 78.7 \\
2 & $5: 1$ & 62.4 & 73.5 \\
3 & $10: 1$ & 44.4 & 70.2 \\
4 & $20: 1$ & 38.5 & 63.9 \\
5 &
\end{tabular}

${ }^{\mathrm{a}}$ Determined by GC analysis. E/S: 5 , temperature: $55^{\circ} \mathrm{C}$.

\section{1.d. Effect of enzyme:substrate ratio}

The influence of the enzyme:substrate ratio was evaluated with a 2.5:1 estradiol/oleic acid ratio varying the amount of CAL. 3,17- $\beta$-Estradiol-17-oleate $\mathbf{3 h}$ formation was then monitored at $72 \mathrm{~h}$ by GC (Figure 2). From the obtained results, it can be concluded that a ratio E/S of 5 is the most satisfactory.

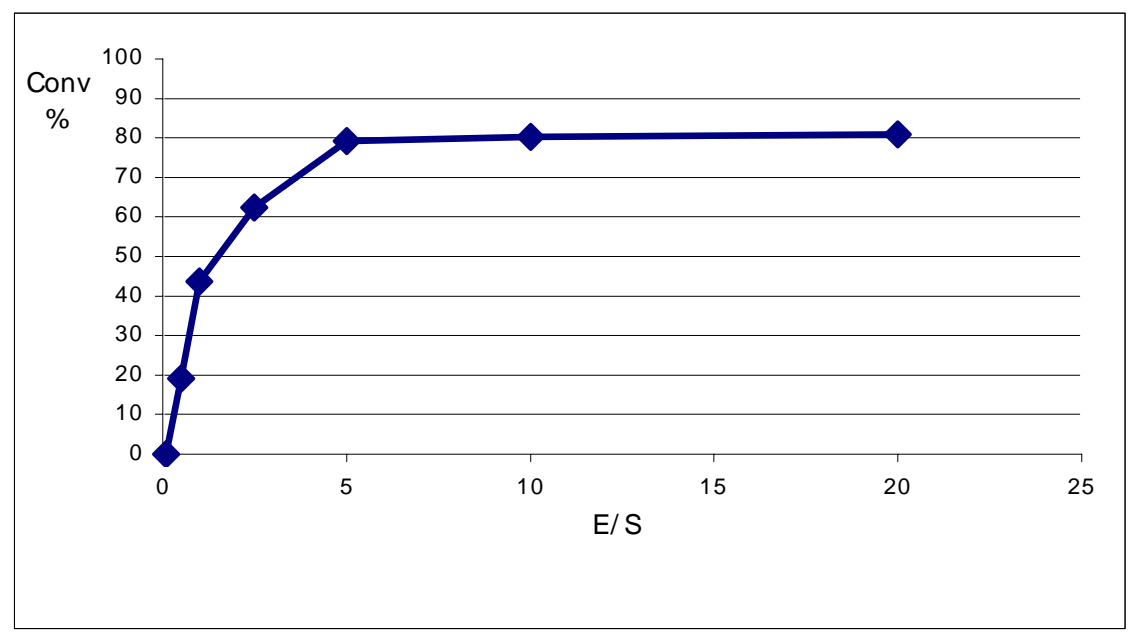

Figure 2. Effect of enzyme:substrate ratio on the CRL-catalyzed synthesis of 3,17- $\beta$-estradiol17-oleate (3h). Acylating agent $/ \mathrm{S}: 2.5$, temperature: $55^{\circ} \mathrm{C}$, time $72 \mathrm{~h}$. 


\section{1.e. Influence of temperature on 3,17- $\beta$-Estradiol-17-oleate formation}

Temperatures of $33^{\circ} \mathrm{C}$ and $55^{\circ} \mathrm{C}$ were considered in reactions where the previously studied parameters were fixed to their optimal values (CRL, toluene, E/S: 5 and oleic acid/estradiol: 2.5). The obtained results: $62 \%$ at $33^{\circ} \mathrm{C}$ and $79 \%$ at $55^{\circ} \mathrm{C}$, showed a difference in terms of yields for the catalyzed reaction at both temperatures. So we decided to perform the reaction at $55^{\circ} \mathrm{C}$.

\section{1.f. Application of the enzymatic acylation to several acylating agents}

The results obtained by means of the CRL catalyzed procedure to the preparation of 17monoacyl fatty acid derivatives of 3,17- $\beta$-estradiol are shown in Table 2. Variable chain length saturated and unsaturated carboxylic acids from 5 to 18 carbon atoms gave the monoacyl derivative exclusively in position 17 in high yield. The results show that the ester formation was not influenced by chain length nor by the presence or stereochemistry of double bonds.

Table 2. Lipase-catalyzed synthesis of 3,17- $\beta$-estradiol-17-esters from carboxylic acids (3)

\begin{tabular}{cccc}
\hline Entry & Product & Acylating agent & Yield (\%) \\
\hline 1 & $\mathbf{3}^{\mathbf{a}}$ & Valeric acid & 67 \\
2 & $\mathbf{3 b}$ & Caproic acid & 66 \\
3 & $\mathbf{3 c}$ & Capric acid & 69 \\
4 & $\mathbf{3 d}$ & Lauric acid & 73 \\
5 & $\mathbf{3 e}$ & Myristic acid & 78 \\
6 & $\mathbf{3 f}$ & Palmitic acid & 70 \\
7 & $\mathbf{3 g}$ & Estearic acid & 71 \\
8 & $\mathbf{3 h}$ & Oleic acid & 76 \\
9 & $\mathbf{3 i}$ & Elaidic acid & 75 \\
10 & $\mathbf{3 j}$ & Linoleic acid & 72 \\
11 & $\mathbf{3 k}$ & Linolenic acid & 77 \\
\hline
\end{tabular}

${ }^{\mathrm{a}}$ Isolated products. E/S: 5, Acylating/S: 2.5 , temperature: $55^{\circ} \mathrm{C}$, time $72 \mathrm{~h}$.

2. Enzymatic alcoholysis

Another approach to obtain 3,17- $\beta$-estradiol-17-oleate $\mathbf{3 h}$ could be an enzymatic alcoholysis on the dioleyl derivative of 3,17- $\beta$-estradiol 4 (Scheme 2). 


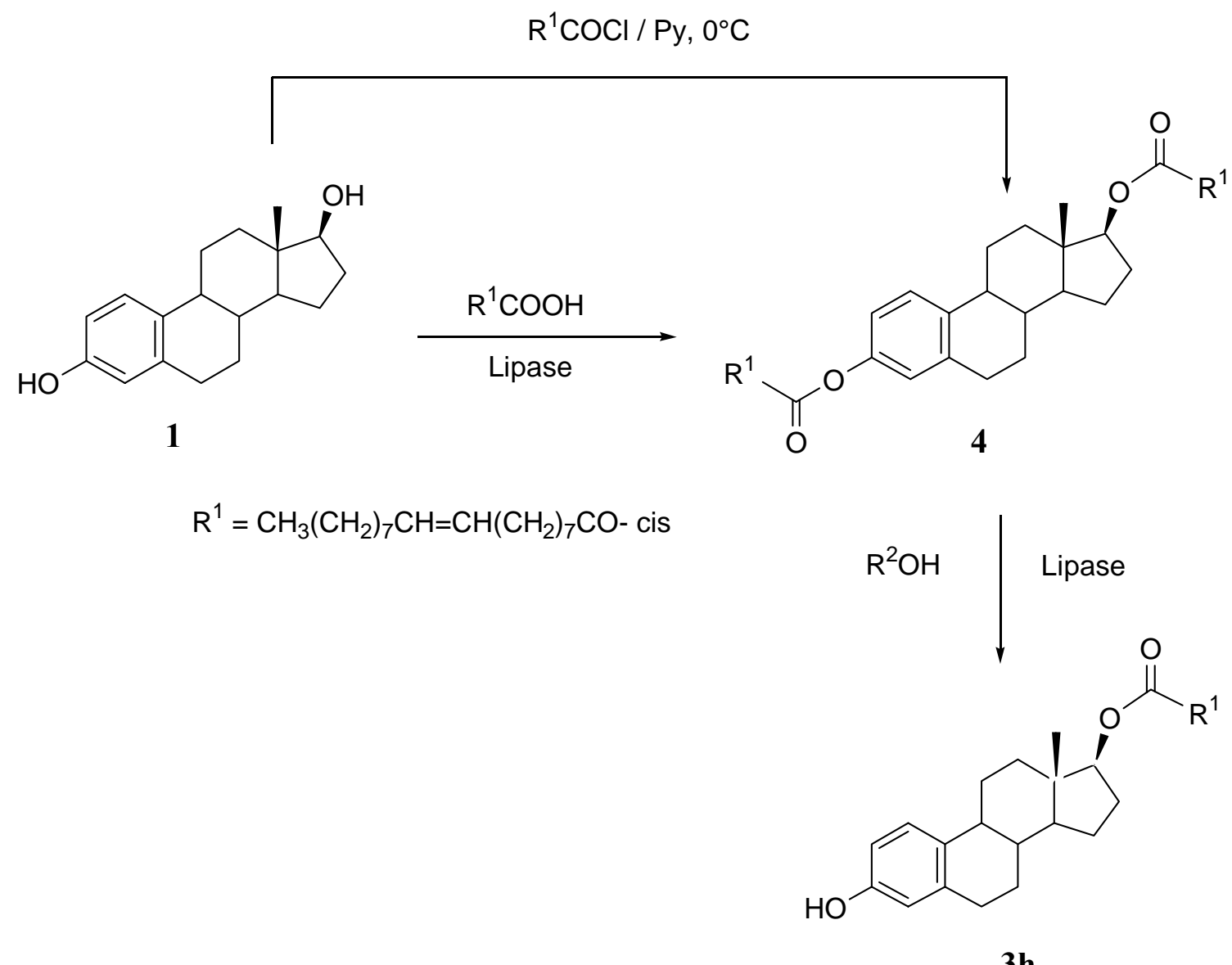

$3 \mathbf{h}$

\section{Scheme 2}

First, we prepared 4, following the reported chemical procedure with oleoyl chloride in pyridine at $0^{\circ} \mathrm{C} .{ }^{27}$ This procedure afforded a mixture of products: a major product which is the 3,17 diester and two minor products corresponding to the C-3 monoester and C-17 monoester, as well as free estradiol. The dioleoyl derivative 4 was isolated and purified by column chromatography. When we tested the performance of lipases in the preparation of 4, we observed that working without solvent at $55^{\circ} \mathrm{C}$, CRL afforded the 3,17-dioleate in high purity and yield $(92 \%)$.

So we scaled up this procedure and then carried out the enzymatic alcoholysis of 3,17- $\beta$ estradiol-dioleate 4. The best enzyme in this case was CAL B. The alcoholysis reaction was conducted with several alcohols as nucleophiles and solvent, leading to similar results with all of them (Table 3). 
Table 3. Lipase-catalyzed alcoholysis of 3,17- $\beta$-estradiol-dioleate (4)

\begin{tabular}{cc}
\hline Alcohol & Yield (\%) \\
\hline Ethanol & 95 \\
Butanol & 94 \\
Octanol & 90 \\
\hline
\end{tabular}

${ }^{\mathrm{a}}$ Determined by GC analysis. E/S: 5 , temperature: $55^{\circ} \mathrm{C}$.

Although this enzymatic approach involving two steps: preparation and alcoholysis of estradiol dioleate 4, gave the products in excellent yield, 92\% and 91\% isolated products respectively, for preparative purposes the acylation procedure to obtain the 17-monoacyl derivatives of 3,17- $\beta$-estradiol seems to be simpler and more convenient.

\section{Conclusions}

Following an enzymatic methodology a series of acyl esters of 3,17- $\beta$-estradiol has been prepared. The 17-monoacyl products were obtained in a high regioselective way by acylation of $3,17-\beta$-estradiol or by alcoholysis of the corresponding diacyl derivatives. Five $(\mathbf{3 b}, \mathbf{3 c}, \mathbf{3 d}, \mathbf{3 e}$ and 3i) from the eleven compounds prepared, have not been described before. The influence of various reaction parameters was evaluated to determine the reaction conditions. Among the tested lipases, lipase from Candida rugosa appeared to be the most appropriate in the acylation reaction CAL B in the alcoholysis. It was observed that in the acylation, the direct esterification with fatty acids gave better results than transesterification with their ethyl esters. The advantages presented by this methodology such as mild reaction conditions, economy and low environmental impact, make the biocatalysis a convenient way to prepare in a regioselective way, monoacyl derivatives of 3,17- $\beta$-estradiol containing the aromatic $3-\mathrm{OH}$ free. Some of these compounds (3a and $\mathbf{3 h}$ ) are recognized as useful products in pharmaceutical industry and belong to the "long acting" estrogens.

\section{Experimental Section}

General Procedures. All solvents and reagents were reagent grade and used without purification. Lipase from Candida rugosa (CRL) (905 U/mg solid), pancreatin and type II crude from porcine pancreas (PPL) (190 U/mg protein) were purchased from Sigma Chemical Co.; Candida antarctica lipase B (CAL B): Novozym 435 (7400 PLU/g) and Lipozyme RM 1M (LIP) $(7800 \mathrm{U} / \mathrm{g})$ were generous gifts of Novozymes Latinoamerica Ltda and Novozymes A/S; Pseudomonas lipase: Lipase PS Amano (PSL) (33,200 U/g) was purchased to Amano Pharmaceutical Co. All enzymes were used "straight from the bottle". 
Enzymatic reactions were carried out on Innova 4000 digital incubator shaker, New Brunswick Scientific Co. at $33^{\circ} \mathrm{C}$ and $55^{\circ} \mathrm{C}$ and $200 \mathrm{rpm}$. Melting points were measured in a Fisher Johns apparatus and are uncorrected. Enzymatic reactions were followed by TLC on Merck Silica gel 60F-254 aluminium sheets (0.2 mm thickness). For column chromatography Merck Silica gel 60 (60-230 mesh) was used. GC analyses were performed on a Hewlett Packard-5890 gas chromatograph, using HP-17 column $(10 \mathrm{mx} 0.53 \mathrm{mmx} 0.20 \mu \mathrm{m})$. The following temperature program was employed $100^{\circ} \mathrm{C}(1 \mathrm{~min}) / 5^{\circ} \mathrm{C} / \mathrm{min} / 280^{\circ} \mathrm{C}(5 \mathrm{~min})$. FT-IR spectra were obtained on a Shimadzu FTIR-8300 spectrophotometer. ${ }^{1} \mathrm{H}-\mathrm{NMR}$ and ${ }^{13} \mathrm{C}-\mathrm{NMR}$ spectra were recorded at $500 \mathrm{MHz}$ using Bruker AM-500 spectrometer. Chemical shifts are reported in $\delta$ units relative to tetramethylsilane (TMS) set at $0 \delta$, using $\mathrm{CDCl}_{3}$ as solvent, coupling constants are given in Hz. EI-MS were obtained at 70eV using a TRIO-2 VG Masslab Shimadzu QP-5000 and a Finnigan TSQ70 instrument mass spectrometers.

\section{Enzymatic monoacylation}

(17ß)-Estra-1,3,5(10)-triene-3,17-diol cis-9-octadecenoate (3h). To a solution of 1 (1 g, 3.7 $\mathrm{mmol})$ in toluene $(300 \mathrm{ml}), 2.6 \mathrm{~g}(9.25 \mathrm{mmol})$ of oleic acid and $5 \mathrm{~g}$ of CRL were added. The suspension was stirred $(200 \mathrm{rpm})$ at $55^{\circ} \mathrm{C}$ and the progress of reaction was monitored by TLC (hexane/dichloromethane 1:3)) and GC. After the indicated time, the enzyme was filtered off, the solvent evaporated, and the crude residue purified by silica gel chromatography (hexane: dichloromethane 1:3): $1.50 \mathrm{~g}(76 \%)$ of 3h. m. p.: 38-41 ${ }^{\circ} \mathrm{C}$. IR $v_{\text {máx }}\left(\mathrm{cm}^{-1}\right) 3467(\mathrm{OH}), 3010$, 2933, $2861(\mathrm{CH}), 1702(\mathrm{C}=\mathrm{O}) .{ }^{1} \mathrm{H}$ NMR $\delta 0.83(\mathrm{~s}, 3 \mathrm{H}), 0.88(\mathrm{t}, J=7.02 \mathrm{~Hz}, 3 \mathrm{H}), 1.57-1.23(\mathrm{~m}$, 28H), 1.67-1.60 (m, 2H), 1.78-1.70 (m, 1H), 1.90-1.84 (m, 2H), 2.02 (dd $J=12.3 \mathrm{~Hz}, 6.5 \mathrm{~Hz}$, $4 \mathrm{H}), 2.29-2.15(\mathrm{~m}, 3 \mathrm{H}), 2.32(\mathrm{t}, J=7.7 \mathrm{~Hz}, 2 \mathrm{H}), 2.85-2.80(\mathrm{~m} 2 \mathrm{H}), 4.70(\mathrm{dd}, J=9.06 \mathrm{~Hz}, 7.95$, $1 \mathrm{H}), 5,35(\mathrm{~m}, 2 \mathrm{H}), 6.57(\mathrm{~d}, J=2.74 \mathrm{~Hz}, 1 \mathrm{H}), 6.64(\mathrm{dd}, J=8.44 \mathrm{~Hz}, 2.75 \mathrm{~Hz}, 1 \mathrm{H}), 7.14(\mathrm{~d}, J=$ $8.35 \mathrm{~Hz}, 1 \mathrm{H}) \mathrm{ppm} .{ }^{13} \mathrm{C} \mathrm{NMR}\left(\mathrm{CDCl}_{3}\right): 12.15,14.2,22.5,23.3,25.2,25.4,25.7,26.2,27.1,27.4$, 27.6, 29.2, 29.3, 29.4, 29.5, 29.6, 29.7, 31.9, 34.7, 36.7, 38.5, 43.2, 49.8, 82.5, $112.7,115.3$, 126.6, 127.4, 127.8, 128.3, 130.4, 131.7, 132.6, 138.2, 153.4, 174.1 ppm. EI-MS: m/z (relative intensity): $536\left[\mathrm{M}^{+}\right](17), 281(2), 255$ (100), 159 (67), 133 (37), 95 (15), 69 (25), 55 (40), 41 $(18) .^{27}$

(17ß)-Estra-1,3,5(10)-triene-3,17-diol pentanoate (3a). As described for $\mathbf{3 h}$, but using pentanoic acid $(940 \mathrm{mg})$ as acylating agent: $877 \mathrm{mg}(67 \%)$, m.p.: $146-7^{\circ} \mathrm{C}\left(\text { lit. } 148{ }^{\circ} \mathrm{C}\right)^{31}$;

IR $v_{\text {máx }}\left(\mathrm{cm}^{-1}\right) 3440(\mathrm{OH}), 3015,2985,2885(\mathrm{CH}), 1700(\mathrm{C}=\mathrm{O}) .{ }^{1} \mathrm{H}-\mathrm{NMR} \delta 0.83(\mathrm{~s}, 3 \mathrm{H}), 0.93$ $(\mathrm{t}, J=7.42 \mathrm{~Hz}, 3 \mathrm{H}), 1.48-122(\mathrm{~m}, 8 \mathrm{H})), 1.58-1.49(\mathrm{~m}, 1 \mathrm{H}), 1.69-1.59(\mathrm{~m}, 2 \mathrm{H}), 1.79-1.66(\mathrm{~m}$, 2H), 1.90-1.82 (m, 2H), 2.30-2.15 (m, 3H), $2.32(\mathrm{t}, J=7.4 \mathrm{~Hz}, 2 \mathrm{H}), 2.81(\mathrm{~m}, 2 \mathrm{H}), 4.70(\mathrm{dd}, J=$ $7.8 \mathrm{~Hz}, 8.0 \mathrm{~Hz}, 1 \mathrm{H}), 6.57(\mathrm{~d}, J=2.7 \mathrm{~Hz}, 1 \mathrm{H}), 6.63(\mathrm{dd}, J=8.2 \mathrm{~Hz}, 2.7 \mathrm{~Hz}, 1 \mathrm{H}), 7.14(\mathrm{~d}, J=8.2$ $\mathrm{Hz}, 1 \mathrm{H}) \mathrm{ppm} .{ }^{13} \mathrm{C}-\mathrm{NMR} \delta 12.1,13.7,22.2,23.2,26.1,27.2,27.3,27.6,29.6,34.4,36.9,38.6$, $42.9,43.7,49.7,82.5,112.7,115.2,126.5,132.5,138.1,153.4,174.1 \mathrm{ppm} . \mathrm{EI} / \mathrm{MS} \mathrm{m} / \mathrm{z}$ (relative intensity): $356 \mathrm{M}^{+}(100), 85$ (63), 57 (81), 41 (42).

(17ß)-Estra-1,3,5(10)-triene-3,17-diol hexanoate (3b). As described for $3 \mathrm{~h}$, but using hexanoic acid (1,08 g) as acylating agent: : $898 \mathrm{mg}(66 \%)$, m.p.: $118-121^{\circ} \mathrm{C}$; IR $v_{\text {máx }}\left(\mathrm{cm}^{-1}\right) 3438(\mathrm{OH})$, 
3018, 2980, $2880(\mathrm{CH}), 1710(\mathrm{C}=\mathrm{O}) .{ }^{1} \mathrm{H}$ NMR $\left(\mathrm{CDCl}_{3}\right) \delta 0.83(\mathrm{~s}, 3 \mathrm{H}), 0 . .81(\mathrm{t}, J=7.03 \mathrm{~Hz}, 3 \mathrm{H})$, $1.40-1.25(\mathrm{~m}, 9 \mathrm{H}), 1.64-1.60(\mathrm{~m}, 4 \mathrm{H}), 1.77-1.71(\mathrm{~m}, 2 \mathrm{H}), 1.89-1.84(\mathrm{~m}, 2 \mathrm{H}), 2.28-2.17(\mathrm{~m}, 3 \mathrm{H})$, $2.31(\mathrm{t}, J=7.85 \mathrm{~Hz}, 2 \mathrm{H}), 2.82(\mathrm{~m}, 2 \mathrm{H}), 4.69(\mathrm{dd}, J=7.85 \mathrm{~Hz}, 9.08 \mathrm{~Hz}, 1 \mathrm{H}), 6.56(\mathrm{~d}, J=2.69 \mathrm{~Hz}$, $1 \mathrm{H}), 6.62(\mathrm{dd}, J=8.41 \mathrm{~Hz}, 2.80 \mathrm{~Hz}, 1 \mathrm{H}), 7.14(\mathrm{~d}, J=8.30 \mathrm{~Hz}, 1 \mathrm{H}) \mathrm{ppm} .{ }^{13} \mathrm{C}$ NMR $\left(\mathrm{CDCl}_{3}\right) \delta$ (ppm) : 12.1, 13.9, 22.3, 23.3, 24.8, 26.2, 27.2, 27.6, 29.6, 31.3, 34.6, 36.9, 38.5, 42.9, 43.8, 49.8, $82.5,112.7,115.2,126.5,132.5,138.2,153.4,174.1 \mathrm{ppm}$. EI-MS $\mathrm{m} / \mathrm{z}$ (relative intensity): 370[M $\left.\mathrm{M}^{+}\right]$(100), 254 (13), 225 (14), 172 (22), 159 (28), 146 (26), 133 (22), 99(28), 71 (33) 55 (20), 43(72). HR-MS: $370.5359\left(\mathrm{C}_{24} \mathrm{H}_{34} \mathrm{O}_{3}{ }^{+}\right.$; calc. 370.5366).

(17ß)-Estra-1,3,5(10)-triene-3,17-diol decanoate (3c). As described for $\mathbf{3 h}$, but using decanoic acid $(1.6 \mathrm{~g})$ as acylating agent: : $1.08 \mathrm{~g}(69 \%)$, m.p.: $94-99^{\circ} \mathrm{C}$; IR $v_{\text {máx }}\left(\mathrm{cm}^{-1}\right) 3450(\mathrm{OH}), 3009$, 2989, $2853(\mathrm{CH}), 1701(\mathrm{C}=\mathrm{O}) .{ }^{1} \mathrm{H}$ NMR $\delta 0.76(\mathrm{~s}, 3 \mathrm{H}), 0.84(\mathrm{t}, J=7.05 \mathrm{~Hz}, 3 \mathrm{H}), 1.44-1.18(\mathrm{~m}$, $17 \mathrm{H}), 1.61-1.54(\mathrm{~m}, 4 \mathrm{H}), 1.71-1.64(\mathrm{~m}, 2 \mathrm{H}), 1.83-1.78(\mathrm{~m}, 2 \mathrm{H}), 2.22-2.11(\mathrm{~m}, 3 \mathrm{H}), 2.25(\mathrm{t}, J=$ $7.65 \mathrm{~Hz}, 2 \mathrm{H}), 2.79-2.73(\mathrm{~m}, 2 \mathrm{H}), 4.64(\mathrm{dd}, J=7.89 \mathrm{~Hz}, 9.09 \mathrm{~Hz}, 1 \mathrm{H}), 6.50(\mathrm{~d}, J=2.66 \mathrm{~Hz}, 1 \mathrm{H})$, $6.56(\mathrm{dd}, J=8.40 \mathrm{~Hz}, 2.83 \mathrm{~Hz}, 1 \mathrm{H}), 7.08(\mathrm{~d}, J=8.48 \mathrm{~Hz}, 1 \mathrm{H}) \mathrm{ppm} .{ }^{13} \mathrm{C}^{\mathrm{NMR}}\left(\mathrm{CDCl}_{3}\right) \delta: 12.1$, 14.1, 22.7, 23.3, 25.1, 26.2, 27.1, 27.6, 29.1, 29.3, 29.4, 29.6, 29.7, 31.8, 34.6, 36.9, 38.5, 42.9, $43.8,49.8,82.4,112.6,115.2,126.5,132.6,138.2,153.3,174.1 \mathrm{ppm}$. EI-MS m/z (relative intensity): $426\left[\mathrm{M}^{+}\right]$(100), 254 (20), 172 (28), 159 (44), 146 (31), 133 (39), 71 (26), 57 (48) 43 (78). HR-MS: $426.6454\left(\mathrm{C}_{28} \mathrm{H}_{42} \mathrm{O}_{3}{ }^{+}\right.$; calc. 426.6450).

(17ß)-Estra-1,3,5(10)-triene-3,17-diol dodecanoate (3d). As described for $\mathbf{3 h}$, but using dodecanoic acid $(1.85 \mathrm{~g})$ as acylating agent: : $1.22 \mathrm{~g}(73 \%)$, m.p.: $70-71^{\circ} \mathrm{C}$; IR $v_{\text {máx }}\left(\mathrm{cm}^{-1}\right) 3460$ $(\mathrm{OH}), 3009,2989,2853(\mathrm{CH}), 1701(\mathrm{C}=\mathrm{O}) .{ }^{1} \mathrm{H}-\mathrm{NMR} \delta 0.83(\mathrm{~s}, 3 \mathrm{H}), 0.88(\mathrm{t}, J=7.45 \mathrm{~Hz}, 3 \mathrm{H})$, 1.48-1.06 (m, 20H) ), 1.58-1.49 (m, 1H), 1.66-1.59 (m, 4H), 1.78-1.69 (m, 2H), 1.90-1.82 (m, 2H), $2.20-2.14(\mathrm{~m}, 3 \mathrm{H}), 2.32(\mathrm{t}, J=7.7 \mathrm{~Hz}, 2 \mathrm{H}), 2.82(\mathrm{~m}, 2 \mathrm{H}), 4.70(\mathrm{dd}, J=7.8 \mathrm{~Hz}, 8.0 \mathrm{~Hz}$, $1 \mathrm{H}), 6.55(\mathrm{~d}, J=2.8 \mathrm{~Hz}, 1 \mathrm{H}), 6.63(\mathrm{dd}, J=8.5 \mathrm{~Hz}, 2.8,1 \mathrm{H}), 7.14(\mathrm{~d}, J=8.2 \mathrm{~Hz}, 1 \mathrm{H}) \mathrm{ppm} .{ }^{13} \mathrm{C}-$ NMR $\delta 12.1,14.1,22.7,23.2,25.1$ 26.2, 27.1, 27.6, 29.1, 29.2, 29.3, 29.4, 29.5, 29.6, 29.7,31.9, 34.6, 36.9, 38.5, 42.9, 43.8, 49.8, 82.5, 112.7, 115.2, 126.5, 132.5, 138.1, 153.8, 174.2 ppm. EI/MS m/z (relative intensity): $454\left[\mathrm{M}^{+}\right]$(100), 255 (15), 159 (31), 57 (37), 43 (56). HR-MS: $454.6995\left(\mathrm{C}_{30} \mathrm{H}_{46} \mathrm{O}_{3}{ }^{+}\right.$; calc. 454.6992).

(17ß)-Estra-1,3,5(10)-triene-3,17-diol tetradecanoate (3e). As described for $3 \mathbf{h}$, but using tetradecanoic acid $(2.1 \mathrm{~g})$ as acylating agent: : $1.38 \mathrm{~g}(78 \%)$, m.p.: $68-72^{\circ} \mathrm{C}$; IR $v_{\text {máx }}\left(\mathrm{cm}^{-1}\right)$ $3431(\mathrm{OH}), 3011,2989,2863(\mathrm{CH}), 1706(\mathrm{C}=\mathrm{O}) .{ }^{1} \mathrm{H}$ NMR $\delta 0.79(\mathrm{~s}, 3 \mathrm{H}), 0.87(\mathrm{t}, J=7.39 \mathrm{~Hz}$, $3 \mathrm{H}), 1.35-1.07(\mathrm{~m}, 24 \mathrm{H}), 1.61-1.47(\mathrm{~m}, 1 \mathrm{H}), 1.65-1.57(\mathrm{~m}, 4 \mathrm{H}), 1.74-1.67(\mathrm{~m}, 2 \mathrm{H}), 1.89-1.79$ (m, 2H), 2.17-2.15 (m, 3H), $2.23(\mathrm{t}, J=7.65 \mathrm{~Hz}, 2 \mathrm{H}), 2.87(\mathrm{~m}, 2 \mathrm{H}), 4.78(\mathrm{dd}, J=7.72 \mathrm{~Hz}, 8.10$ $\mathrm{Hz}, 1 \mathrm{H}), 6.55(\mathrm{~d}, J=2.72 \mathrm{~Hz}, 1 \mathrm{H}), 6.63(\mathrm{dd}, J=8.48 \mathrm{~Hz}, 2.71,1 \mathrm{H}), 7.14(\mathrm{~d}, J=8.19 \mathrm{~Hz}, 1 \mathrm{H})$ ppm. ${ }^{13} \mathrm{C}$ NMR $\delta 12.1,14.1,22.7,23.3,24.8,26.2,27.1,27.6,29.1,29.2,29.4,29.5,29.6,29.7$, 29.7, 31.3, 34.6, 36.9, 38.5, 42.9, 43.8, 49.8, 82.5, 112.7, 115.2, 126.5, 132.5, 138.2, 153.4, 174.1 ppm. EI-MS m/z (relative intensity): $482\left[\mathrm{M}^{+}\right]$(52), 255 (13), 172 (14), 159 (38), 146 (24), 133 (23) , 71 (16), 43 (100). HR-MS: $482.7529\left(\mathrm{C}_{32} \mathrm{H}_{50} \mathrm{O}_{3}{ }^{+}\right.$; calc. 482.7532$)$.

(17ß)-Estra-1,3,5(10)-triene-3,17-diol hexadecanoate (3f). As described for $\mathbf{3 h}$, but using hexadecanoic acid $(2.4 \mathrm{~g})$ as acylating agent: : $1.31 \mathrm{~g}(70 \%)$, m.p.: $71-73^{\circ} \mathrm{C}$. IR $v_{\text {máx }}\left(\mathrm{cm}^{-1}\right) 3423$ 
$(\mathrm{OH}), 3015,2990,2871(\mathrm{CH}), 1701(\mathrm{C}=\mathrm{O}) .{ }^{1} \mathrm{H}$ NMR $\delta 0.82(\mathrm{~s}, 3 \mathrm{H}), 0.88(\mathrm{t}, J=6.94 \mathrm{~Hz}, 3 \mathrm{H})$, $1.55-1.22(\mathrm{~m}, 3 \mathrm{H}), 1.65-1.22(\mathrm{~m}, 4 \mathrm{H}), 1.78-1.70(\mathrm{~m}, 1 \mathrm{H}), 1.90-1.84(\mathrm{~m}, 2 \mathrm{H}), 2.26-2.15(\mathrm{~m}, 3 \mathrm{H})$, $2.31(\mathrm{t}, J=7.83 \mathrm{~Hz}, 3 \mathrm{H}), 2.85-2.80(\mathrm{~m}, 1 \mathrm{H}), 4.69(\mathrm{dd}, J=7.93 \mathrm{~Hz}, 9.10 \mathrm{~Hz}, 1 \mathrm{H}), 6.56(\mathrm{~d}, J=$ $2.67 \mathrm{~Hz}, 1 \mathrm{H}), 6.62(\mathrm{dd}, J=8.42 \mathrm{~Hz}, 2.77 \mathrm{~Hz}, 1 \mathrm{H}), 7.14(\mathrm{~d}, J=8.32 \mathrm{~Hz}, 1 \mathrm{H}) \mathrm{ppm} .{ }^{13} \mathrm{C} \mathrm{NMR} \delta$ 12.1 , 14.2, 23.3, 25.2, 26.2, 27.2, 27.6, 29.2, 29.3, 29.4, 29.5, 29.6, 29.6, 29.7, 29.7, 31.9, 34.7, $36.9,38.6,43.0,43.8,49.8,82.5,112.7,115.3,126.6,132.6,138.2,153.4,174.1$ ppm. EI-MS $\mathrm{m} / \mathrm{z}$ (relative intensity): $510\left[\mathrm{M}^{+}\right]$(50), 255 (13), 172 (14), 159 (34), 146 (21), 133 (28) ,71 (16), $43(100)^{27}$

(17ß)-Estra-1,3,5(10)-triene-3,17-diol octadecanoate (3g). As described for $\mathbf{3 h}$, but using octadecanoic acid $(2.63 \mathrm{~g})$ as acylating agent: : $1.40 \mathrm{mg}(71 \%)$, m.p.: $65-67^{\circ} \mathrm{C}$. IR $v_{\text {máx }} 3439$ $(\mathrm{OH}), 3011,2983,2851(\mathrm{CH}), 1708(\mathrm{C}=\mathrm{O}) .{ }^{1} \mathrm{H}-\mathrm{NMR} \delta 0.83(\mathrm{~s}, 3 \mathrm{H}), 0.88(\mathrm{t}, J=7.0 \mathrm{~Hz}, 3 \mathrm{H})$, $1.48-1.20(\mathrm{~m}, 32 \mathrm{H})), 1.65-1.49(\mathrm{~m}, 5 \mathrm{H}), 1.77-1.70(\mathrm{~m}, 2 \mathrm{H}), 1.90-1.81(\mathrm{~m}, 2 \mathrm{H}), 2.28-2.16(\mathrm{~m}$, $3 \mathrm{H}), 2.31(\mathrm{t}, J=7.1 \mathrm{~Hz}, 2 \mathrm{H}), 2.82(\mathrm{~m}, 2 \mathrm{H}), 4.70(\mathrm{dd}, J=7.6 \mathrm{~Hz}, 7.9 \mathrm{~Hz}, 1 \mathrm{H}), 6.55(\mathrm{~d}, J=2.9$ $\mathrm{Hz}, 1 \mathrm{H}), 6.63(\mathrm{dd}, J=8.3 \mathrm{~Hz}, 2.9 \mathrm{~Hz}, 1 \mathrm{H}), 7.14$ (d, $J=8.3 \mathrm{~Hz}, 1 \mathrm{H}) \mathrm{ppm} .{ }^{13} \mathrm{C}-\mathrm{NMR} \delta 12.1,14.2$, 22.7, 23.2, 25.2 26.3, 27.2, 27.6, 29.2, 29.3, 29.4, 29.5, 29.6, 29.7, 29.8, 32.0, 34.7, 37.0, 38.6, 43.0, 43.8, 49.8, 82.5, 112.7, 115.3, 126.6, 132.6, 138.2, 153.4, $174.1 \mathrm{ppm}$. EI/MS m/z (relative intensity): $538\left[\mathrm{M}^{+}\right](100), 255$ (35), $159(60), 57$ (53), 43 (78). ${ }^{27}$

(17ß)-Estra-1,3,5(10)-triene-3,17-diol trans-9-octadecenoate (3i). As described for $\mathbf{3 h}$, but using trans-9-ocatadecenoic acid (2.6 g) as acylating agent: : $1.48 \mathrm{~g}$ ( $75 \%$ ), m.p.: 60-64 ${ }^{\circ} \mathrm{C}$; IR $v_{\text {máx }}\left(\mathrm{cm}^{-1}\right) 3429(\mathrm{OH}), 3000,2983,2850(\mathrm{CH}), 1710(\mathrm{C}=\mathrm{O}) .{ }^{1} \mathrm{H}$ NMR $\delta 0.82(\mathrm{~s}, 3 \mathrm{H}), 0.87(\mathrm{t}, J=$ $6.95,3 \mathrm{H}), 1.56-1.23(\mathrm{~m}, 28 \mathrm{H}), 1.65-1.61(\mathrm{~m}, 2 \mathrm{H}), 1.78-1.69(\mathrm{~m}, 1 \mathrm{H}), 1.90-1.83(\mathrm{~m}, 2 \mathrm{H}), 2.01$ $(\mathrm{dd} J=12.3,6.54 \mathrm{H}), 2.30-2.16(\mathrm{~m}, 3 \mathrm{H}), 2.31(\mathrm{t}, J=7.60 \mathrm{~Hz}, 2 \mathrm{H}), 2.85-2.80(\mathrm{~m} \mathrm{2H}), 4.70(\mathrm{dd}, J$ $=9.06 \mathrm{~Hz}, 7.81 \mathrm{~Hz}, 1 \mathrm{H}), 5,35(\mathrm{~m}, 2 \mathrm{H}), 6.57(\mathrm{~d}, J=2.74 \mathrm{~Hz}, 1 \mathrm{H}), 6.65(\mathrm{dd}, J=8.37 \mathrm{~Hz}, 2.79 \mathrm{~Hz}$, $1 \mathrm{H}), 7.14(\mathrm{~d}, J=8.37 \mathrm{~Hz}, 1 \mathrm{H}) \mathrm{ppm} .{ }^{13} \mathrm{C} \mathrm{NMR}\left(\mathrm{CDCl}_{3}\right): 12.1,14.2,22.7,23.3,25.1,25.5,25.6$, 26.3, 27.2, 27.3, 27.6, 29.1, 29.2, 29.4, 29.6, 29.7, 29.7, 31.9, 33.8, 36.9, 38.6, 43.8, 49.8, 82.5, $112.8,115.3,126.6,127.2,127.6,128.3,130.3,131.0131 .9,138.2,153.4,174.1$ ppm. EI-MS $\mathrm{m} / \mathrm{z}$ (relative intensity): $536\left[\mathrm{M}^{+}\right](20), 255$ (100), 159 (74), 133 (37), 95 (13), 69 (21), 55 (43), 41 (29). HR-MS: $536.8450\left(\mathrm{C}_{36} \mathrm{H}_{56} \mathrm{O}_{3}{ }^{+}\right.$; calc. 536.8459).

(17ß)-Estra-1,3,5(10)-triene-3,17-diol cis,cis-9,12-octadecadienoate (3j). As described for $\mathbf{3 h}$, but using cis,cis-9,12-octadecadienoic acid (2.6 g) as acylating agent: $1.41 \mathrm{~g}$ ( 72\%). m.p.: 35$37^{\circ} \mathrm{C}$. IR $v_{\text {máx }}\left(\mathrm{cm}^{-1}\right) 3414(\mathrm{OH}), 3005,2981,2849(\mathrm{CH}), 1703(\mathrm{C}=\mathrm{O}) .{ }^{1} \mathrm{H}-\mathrm{NMR} \delta 0.83(\mathrm{~s}, 3 \mathrm{H})$, $0.88(\mathrm{t}, J=7.0 \mathrm{~Hz}, 3 \mathrm{H}), 1.48-1.18(\mathrm{~m}, 13 \mathrm{H})), 1.57-1.39(\mathrm{~m}, 3 \mathrm{H}), 1.67-1.58(\mathrm{~m}, 5 \mathrm{H}), 1.78-1.69$ (m, 2H), 1.90-1.82 (m, 2H), 2.11-1.92(m,4H,) 2.28-2.12 (m, 3H), $2.31(\mathrm{t}, J=7.2 \mathrm{~Hz}, 2 \mathrm{H}), 2.1$ $(\mathrm{m}, 4 \mathrm{H}), 4.70(\mathrm{dd}, J=7.9 \mathrm{~Hz}, 7.9 \mathrm{~Hz}, 1 \mathrm{H}), 5.43-5.27(\mathrm{~m}, 4 \mathrm{H}), 6.55$ (d, J=2.5 Hz, 1H), 6.63 (dd, $J=8.3 \mathrm{~Hz}, 2.5 \mathrm{~Hz}, 1 \mathrm{H}), 7.14(\mathrm{~d}, J=8.3 \mathrm{~Hz}, 1 \mathrm{H}) \mathrm{ppm} .{ }^{13} \mathrm{C}-\mathrm{NMR} \delta 12.1,14.2,22.7,23.3,25.1$, 25.5, 25.6, 26.3, 27.2, 27.3, 27.6, 29.1, 29.2, 29.4, 29.6, 29.7, 29.8, 31.9, 34.6, 36.9, 38.6, 43.8, $49.8,82.5,112.8,115.3,126.6,127.2,127.6,128.3,130.3,132.0132 .5,138.2,153.4,174.1$ ppm. EI/MS m/z (relative intensity): $532\left[\mathrm{M}^{+}\right]$(100), 255 (35), 133 (29), 55 (48), 41 (67). ${ }^{27}$

3,17- $\mathbf{\beta}$-Estradiol-17-cis,cis,cis-9,12,15-octadecatrienoate (3k). As described for $\mathbf{3 h}$, but using cis,cis,cis-9,12,15-octadecatrienoic acid (2.6 g) as acylating agent: $1.51 \mathrm{~g}(77 \%)$. Oil. IR $v_{\text {máx }}$ 
$\left(\mathrm{cm}^{-1}\right) 3433(\mathrm{OH}), 3010,2980,2846(\mathrm{CH}), 1705(\mathrm{C}=\mathrm{O}) .{ }^{1} \mathrm{H}-\mathrm{NMR} \delta 0.83(\mathrm{~s}, 3 \mathrm{H}), 0.88(\mathrm{t}, J=7.0$ $\mathrm{Hz}, 3 \mathrm{H}), 1.48-1.18(\mathrm{~m}, 11 \mathrm{H})), 1.57-1.39(\mathrm{~m}, 3 \mathrm{H}), 1.67-1.58(\mathrm{~m}, 5 \mathrm{H}), 1.78-1.69(\mathrm{~m}, 2 \mathrm{H}), 1.90-1.82$ (m, 2H), 2.11-1.92(m,4H,) 2.28-2.12 (m, 3H), 2.31 (t, $J=7.2 \mathrm{~Hz}, 2 \mathrm{H}), 2.1(\mathrm{~m}, 4 \mathrm{H}), 4.70$ (dd, $J$ $=7.9 \mathrm{~Hz}, 7.9 \mathrm{~Hz}, 1 \mathrm{H}), 5.43-5.27(\mathrm{~m}, 6 \mathrm{H}), 6.55(\mathrm{~d}, J=2.5 \mathrm{~Hz}, 1 \mathrm{H}), 6.63(\mathrm{dd}, J=8.3 \mathrm{~Hz}, 2.5 \mathrm{~Hz}$, $1 \mathrm{H}), 7.14(\mathrm{~d}, J=8.3 \mathrm{~Hz}, 1 \mathrm{H}) \mathrm{ppm} .{ }^{13} \mathrm{C}-\mathrm{NMR} \delta 12.1,14.2,22.7,23.3,25.1,25.5,25.6,26.3$, 27.2, 27.3 , 27.6, 29.1, 29.2, 29.4, 29.6, 29.7, 29.8, 31.9, 34.6, 36.9, 38.6, 43.8, 49.8, 82.5, 112.8, $115.3,126.6,127.2,127.6,128.3,130.3,132.0132 .5,138.2,153.4,174.1 \mathrm{ppm} . \mathrm{EI} / \mathrm{MS} \mathrm{m} / \mathrm{z}$ (relative intensity): $532\left[\mathrm{M}^{+}\right](100), 255(35), 133(29), 55(48), 41(67){ }^{32}$

\section{3,17- $\beta$-Estradiol-cis-9-dioctadecenoate (4)}

\section{Chemical synthesis}

It was prepared as described in reference 27 with $10 \mathrm{~g}$ of 3,17- $\beta$-estradiol. The dioleate 4 was isolated from the mixture of products by column chromatography (hexane/dichloromethane 1:3). Yield: $6.8 \mathrm{~g}$ (57\%). Oil. IR $v_{\operatorname{máx}}\left(\mathrm{cm}^{-1}\right)$ 3007, 2990, $2835(\mathrm{CH}), 1735,1702(\mathrm{C}=\mathrm{O}) .{ }^{1} \mathrm{H}-\mathrm{NMR} 0.83$ $(\mathrm{s}, 3 \mathrm{H}), 0.88(\mathrm{t}, J=6.9 \mathrm{~Hz}, 6 \mathrm{H}), 1.58-1.20(\mathrm{~m}, 44 \mathrm{H})), 1.67-1.60(\mathrm{~m}, 6 \mathrm{H}), 1.78-1.70(\mathrm{~m}, 2 \mathrm{H})$, 1.90-1.82 (m, 2H), 2.11-1.95(m, 8H,) 2.28-2.20 (m, 3H), $2.31(\mathrm{t}, J=7.0 \mathrm{~Hz}, 4 \mathrm{H}), 2.89-2.83(\mathrm{~m}$, 2H). $4.70(\mathrm{dd}, J=7.9 \mathrm{~Hz}, 7.9 \mathrm{~Hz}, 1 \mathrm{H}), 5.39-5.30(\mathrm{~m}, 4 \mathrm{H}), 6.78(\mathrm{~d}, J=2.5 \mathrm{~Hz}, 1 \mathrm{H}), 6.82(\mathrm{dd}, J=$ $8.5 \mathrm{~Hz}, 2.5 \mathrm{~Hz}, 1 \mathrm{H}), 7.27$ (d, $J=8.5 \mathrm{~Hz}, 1 \mathrm{H}) \mathrm{ppm} .{ }^{13} \mathrm{C}-\mathrm{NMR} \delta$ 12.1, 14.1, 21.1, 22.7, 23.2, 25.0, 25.1, 26.1, 27.0, 27.1, 27.2, 27.6, 29.0, 29.1, 29.2, 29.3, 29.4, 29.5, 29.6, 29.7, 29.8, 31.9, 34.4, 34.6, 36.9, 38.2, 43.0, 44.0, 49.8, 82.5, 118.6, 121.4, 126.3, 129.6, 129.7, 129.9, 130.0, 137.7, 138.1, 148.5, 172.5, 173.9 ppm. EI/MS m/z (relative intensity): 535 (25), 279 (15), 133 (29), 55 (100), 41 (43).

\section{Enzymatic synthesis}

To a solution of 1 ( $1 \mathrm{~g}, 3.7 \mathrm{mmol}), 9.8 \mathrm{~g}$ (34.5 mmol) of oleic acid and $5 \mathrm{~g}$ of CRL were added. The suspension was stirred $(200 \mathrm{rpm})$ at $55^{\circ} \mathrm{C}$ and the progress of reaction was monitored by TLC (hexane/dichloromethane 1:3) and GC. After 72 h, $100 \mathrm{ml}$ of dichloromethane were added and the enzyme was filtered off. The solvent was evaporated, and the crude residue was purified by silica gel chromatography (hexane/dichloromethane 1:3): $2.7 \mathrm{~g}$ (92\%) of 4. Spectroscopic data were in accordance with $\mathbf{4}$ obtained by chemical synthesis.

\section{Enzymatic alcoholysis of 3,17- $\beta$-estradiol-cis-9-dioctadecenoate (4)}

To a solution of $4(500 \mathrm{mg}(0.62 \mathrm{mmol}))$ in $10 \mathrm{ml}$ of alcohol, $2.5 \mathrm{~g}$ of CAL B were added. The suspension was shaken $(200 \mathrm{rpm})$ at $55^{\circ} \mathrm{C}$ and the progress of reaction was monitored by GC. After $72 \mathrm{~h}$, the enzyme was filtered off, the solvent evaporated, and the crude residue was purified by silica gel chromatography (hexane:dichloromethane 1:3): $316 \mathrm{mg}$ (95\%) of $3 \mathbf{h}$ Spectroscopic data were in accordance with $\mathbf{3 h}$ obtained by enzymatic acylation of $\mathbf{1}$. 


\section{Acknowledgements}

We thank UBA (project X089), ANPCyT (project PICT 06-08293) for partial financial support. The authors gratefully acknowledge M. M Rivero for his assistance in GC analysis.

\section{References and Notes}

1. Ayres, S.; Tang, M.; Subbiah, M. T. J. Lab. Clin. Med. 1996, 128, 367.

2. Rifici, V.A.; Kahchadurian, A.K. Metabolism 1992, 41, 1110.

3. Maziere, C.; Auclair, M.; Ronvezux, M. F. Atherosclerosis 1991, 89, 175.

4. Nakano, M.; Sugioka, K.; Naito, I.; Takekoshi, S.; Niki, E. Biochem. Biophys. Res. Commun. 1987, 142, 919.

5. Ruiz-Larrea, M. B.; Leal, A. M.; Liza, M.; Lacort, M.; de Groot, H. Steroids 1994, 59, 383.

6. Miller, C. P.; Jirkovsky, I.; Hayhurst, D. A.; Adelman, S. J. Steroids 1996, 61, 305.

7. Mukai, K.; Daifuku, K.; Yokohama, S.; Nakano, M. Biochim. Biophys. Acta 1990, 1035, 348.

8. Tikkanen, M. J.; Adlercreutz, H. Biochem. Pharmacol. 2000, 60, 1.

9. Jones, D. L.; James, V. H. T. J. Steroid Biochem. 1995, 22, 243.

10. Larner, J. M.; Pahuja, S. L.; Shackleton, C.H. J. Biol. Chem. 1993, 268, 13893.

11. Hochberg, R. B. Endocr. Rev 1998, 19, 331.

12. Larner, J.; Shackleton, C.; Roitman, E.; Schwartz, P.; Hochberg, R. J. Clin. Endocrinol. Metab 1992, 75, 195.

13. Bommarius, A. S.; Riebel, B. R. In Biocatalysis, Fundamentals and Applications: WileyVCH: Weinheim, 2004; pp 339-372.

14. Saha, B. C.; Demirjian, D.C. In Applied Biocatalysis in Specialty Chemicals and Pharmaceuticals. ACS: Washington DC, 2000; pp 263-273.

15. Gotor, V. Org. Process. Res. Dev. 2002, 6, 420.

16. Carrea, G.; Riva, S. Angew. Chem., Int. Ed. 2000, 39, 2226.

17. Ferrero, M.; Gotor, V. In Stereoselective Biocatalysis; Patel, R. M., Ed; Marcel Dekker: New York, 2000; pp 579-631.

18. Cruz Silva, M. M.; Riva, S.; Sá e Melo, M. L. Tetrahedron 2005, 61, 3065.

19. Secundo, F.; Carrea, G.; De Amici, M.; Joppolo di Ventimiglia, S.; Dordick, J. S. Biotechnol. Bioeng. 2003, 81, 391.

20. Bertinotti, A.; Carrea, G.; Ottolina, G.; Riva, S. Tetrahedron 1994, 50, 13165.

21. Cruz Silva, M. M.; Sá e Melo, M. M.; Parolin, M.; Tessaro, D.; Riva, S.; Danieli, B. Tetrahedron: Asymmetry 2004, 15, 21.

22. Baldessari, A.; Maier, M.S.; Gros, E. G. Tetrahedron Lett. 1995, 36, 4349.

23. Brutomesso, A.C.; Tiscornia, A.; Baldessari, A. Biocatal. \& Biotransf. 2004, 22, 215.

24. Baldessari, A.; Bruttomeso, A.C.; Gros, E. G. Helv. Chim. Acta 1996, 79, 999. 
25. Brutomesso, A.C.; Baldessari, A. J. Mol. Catal. B: Enzym. 2004, 29, 149.

26. Zhongyu, J.; Haizhen, L. Kexue Tongbao (Engl. Trans1.) 1982, 27, 543.

27. Mellon-Nussbaum, S.; Ponticorvo, L.; Schatz, F.; Hochberg, R.B. J. Biol. Chem. 1982, 257, 5678.

28. Baldessari, A.; Mangone, C.P. Biocatal. Biotransform. 2002, 20, 275.

29. Weber, N.; Weitkamp, P.; Mukherjee, K. D. J. Agric. Food Chem. 2001, 49, 67.

30. Villeneuve, P.; Turon, F.; Caro, Y.; Escoffier, R.; Baréa, B.; Barouh, B.; Lago, R.; Piombo, G.; Pina, M. Enzyme Microb. Technol. 2005, 37, 150.

31. Grady, L.T.; Hays, S. E.; King, R. H.; Klein, H. R.; Mader, W. J.: Wyatt, D. K.; Zimmere, R. O. J. Pharm. Sci. 1973, 62, 459.

32. Roy, R.; Belanger, A. J. Steroid. Biochem. 1989, 33, 257. 